\title{
Hyperuricemia prevalence and its association with metabolic disorders: a multicenter retrospective real-world study in China
}

\author{
Shuguang Pang ${ }^{1,2}$, Qiang Jiang ${ }^{1}$, Pei Sun ${ }^{1}$, Yi Li ${ }^{3}$, Yanhua Zhu ${ }^{3}$, Jin Liu ${ }^{3}$ Xiaoran Ye ${ }^{4}$ Ting Chen ${ }^{4}$, \\ Fei Zhao ${ }^{5}$, Wenjun Yang ${ }^{6}$ \\ ${ }^{1}$ Department of Endocrinology, Jinan Central Hospital, Cheeloo College of Medicine, Shandong University, Jinan, China; ${ }^{2}$ Department of \\ Endocrinology, Jinan Central Hospital Affiliated to Shandong First Medical University, Jinan, China; Jiangsu Hengrui Pharmaceuticals Co., Ltd., \\ Lianyungang, China; ${ }^{4}$ Shanghai Palan DataRx Co., Ltd., Shanghai, China; ${ }^{5}$ Shandong Health Medical Big Data Co., Ltd., Jinan, China; ${ }^{6}$ Department \\ of Endocrinology, The Affiliated Hospital of Shandong University of TCM, Jinan, China \\ Contributions: (I) Conception and design: S Pang, Y Li, W Yang; (II) Administrative support: S Pang, W Yang; (III) Provision of study materials or \\ patients: P Sun, Q Jiang, F Zhao; (IV) Collection and assembly of data: J Liu, X Ye; (V) Data analysis and interpretation: T Chen, Y Zhu, W Yang; (VI) \\ Manuscript writing: All authors; (VII) Final approval of manuscript: All authors. \\ Correspondence to: Wenjun Yang. No. 42, Wenhua West Road, Jinan, China. Email: yangwj1972@163.com.
}

\begin{abstract}
Background: The prevalence of hyperuricemia (HUA) and gout continues to increase in China. Research suggests that HUA may be related to many diseases other than gout. However, further population research is required to investigate the association between HUA and metabolic syndromes. This study sought to investigate the prevalence of HUA in an average population in China, and the association between serum uric acid (UA) levels and related metabolic disorders.

Methods: This multicenter retrospective real-world study examined the hospital information system data of 4 tertiary hospitals in 3 provinces in China. The data of patients aged between 18 and 80 years, who had attended at least 1 medical appointment at which their UA level was recorded, were analyzed to evaluate associations between UA levels and metabolic disorders.
\end{abstract}

Results: Among the 374,506 enrolled subjects (49.7\% male; mean age 51.5 years old), the overall prevalence of HUA and gout were $14.8 \%$ and $0.5 \%$, respectively. The prevalence was higher among males than females $(17.6 \%$ vs. $12.0 \%, 0.8 \%$ vs. $0.1 \%$; both $\mathrm{P}<0.001)$. Groups exhibiting higher UA levels had increased adjusted odds ratios for dyslipidemia and chronic kidney disease (CKD) in both sexes. Changes in UA levels from the baseline were negatively correlated with changes in the estimated glomerular filtration rate and hemoglobin A1c among both sexes (all $\mathrm{P}<0.001$ ), and were positively correlated with changes in total cholesterol (TC), triglyceride (TG), and low-density lipoprotein cholesterol (LDL-C) (all $\mathrm{P}<0.05)$ among males, and changes in TC, TG, LDL-C and glucose (all $\mathrm{P}<0.001$ ) among females.

Conclusions: HUA is associated with dyslipidemia and CKD both cross-sectionally and longitudinally. Similar phenomena were observed in both sexes.

Keywords: Hyperuricemia (HUA); serum uric acid level; metabolic disorder; sex difference

Submitted Aug 17, 2021. Accepted for publication Oct 22, 2021.

doi: $10.21037 / \mathrm{atm}-21-5052$

View this article at: https://dx.doi.org/10.21037/atm-21-5052

\section{Introduction}

Hyperuricemia (HUA) is considered the main cause of gout owing to the accumulation of uric acid crystals (1). According to epidemiological studies, HUA may be related to obesity caused by a diet rich in purine, alcohol, meat, and soft drinks (2,3). In recent decades, HUA has become a common metabolic disorder worldwide (4). A meta-analysis indicates that the pooled prevalence of HUA was $13.3 \%$ in mainland China from 2000 to 2014 (5). Another survey showed that the prevalence of HUA was higher in southern 
(19.9\% for men and $9.3 \%$ for women) and rural (20.1\% for men and $9.0 \%$ for women) areas than northern $(17.0 \%$ for men and $6.7 \%$ for women) and urban (16.4\% for men and $6.6 \%$ for women) areas (6).

Serum uric acid (UA) is known to be associated with cardiovascular, kidney and metabolic diseases and its components such as hyperglycaemia, hypertriglyceridaemia and obesity (7). Lipid has been found to have a stronger association with UA than any other metabolic syndrome components, but the role of a single lipid species associated with UA levels was found to vary in different populations (8-10). According to previous research, in both sexes, serum triglyceride (TG) has the strongest association with HUA in the Chinese population (11). Individuals with higher levels of UA are at a higher future risk of type 2 diabetes independent of other known risk factors $(12,13)$. Previous studies have confirmed that insulin resistance exists in gout patients (14). However, UA is negatively correlated with hemoglobin A1c (HbA1c) in type 2 diabetes patients, and positively correlated with $\mathrm{HbA} 1 \mathrm{c}$ in normal glucose serum (15). HUA is also associated with hypertension in a certain Chinese population (16).

Additionally, UA is an important biomarker and a potentially treatable risk factor for cardiovascular diseases (CVDs) (7). Increased uric acid levels appear to be associated with an increased incidence of acute myocardial infarction, stroke and, chronic heart failure in middle-aged subjects with prior CVD $(17,18)$. It will assist readers if this is stated. U-shaped association between uric acid levels and cardiovascular mortality exists in both women and men (19), which may be due to the protective role of uric acid as an antioxidant (20).

Data on the association between HUA and metabolic syndromes in the Chinese population is limited $(6,21,22)$. This study sought to investigate associations among UA and related diseases using Chinese hospital data from urban areas in 3 provinces and to explore the sex-specific association of serum uric acid dynamics with the incidence of metabolismrelated diseases and biochemical measurements.

We present the following article in accordance with the STROBE reporting checklist (available at https://dx.doi. org/10.21037/atm-21-5052).

\section{Methods}

\section{Study design}

A multicenter, retrospective, cross-sectional, real-world study was conducted. The prevalence of HUA and gout overall and in male and female populations was analyzed. The factors affecting differences in prevalence between males and females were also investigated. In addition, associations among UA and metabolism-related diseases and metabolism-related biochemical measurements were assessed. Associations between changes in UA levels from the baseline and changes in metabolism-related biochemical measurements from the baseline were also examined.

The enrolled subjects were categorized using prespecified sex-specific cut-off values for UA levels (male cut-off: 7, 8, and $9 \mathrm{mg} / \mathrm{dL}$; female cut-off: 6,7 , and $8 \mathrm{mg} / \mathrm{dL}$ ). The lowest cut-off values were set as the top values of the normal range for UA in both sexes, and the higher cut-off represented every $1 \mathrm{mg} / \mathrm{dL}$ increase of UA level (23). The index medical appointment at which the UA measurement of each patient was taken was set as the baseline for the cross-sectional analysis. Using the index visit and the follow-up visit data of the participants, a retrospective cohort was established.

\section{Setting}

This study was based on de-identified hospital information system (HIS) data collected from 4 tertiary hospitals in 3 provinces of China from July 2012 to January 2018.

\section{Participants}

A total of 432,002 patients, who had attended at least 1 medical appointment at which their UA level was recorded, were screened. After excluding those with missing date of birth or sex data $(\mathrm{N}=85)$, those aged $<18$ years $(\mathrm{N}=35,392)$, and those aged $>80$ years $(\mathrm{N}=22,019), 374,506$ adult participants were identified and enrolled in this study (see Figure 1). Of these, there were data of at least 1 follow-up UA level (after the baseline level) for 114,054 participants. These data were studied to examine correlations among UA changes and metabolism-related biomarker changes. All procedures performed in this study involving human participants were in accordance with the Declaration of Helsinki (as revised in 2013). The study was approved by committee ethics board of Jinan Central Hospital, Cheeloo College of Medicine, Shandong University, Jinan, China (No. 2020-026-01). Individual consent for this retrospective analysis was waived.

\section{Variable}

In this study, prevalence was defined as the proportion of 


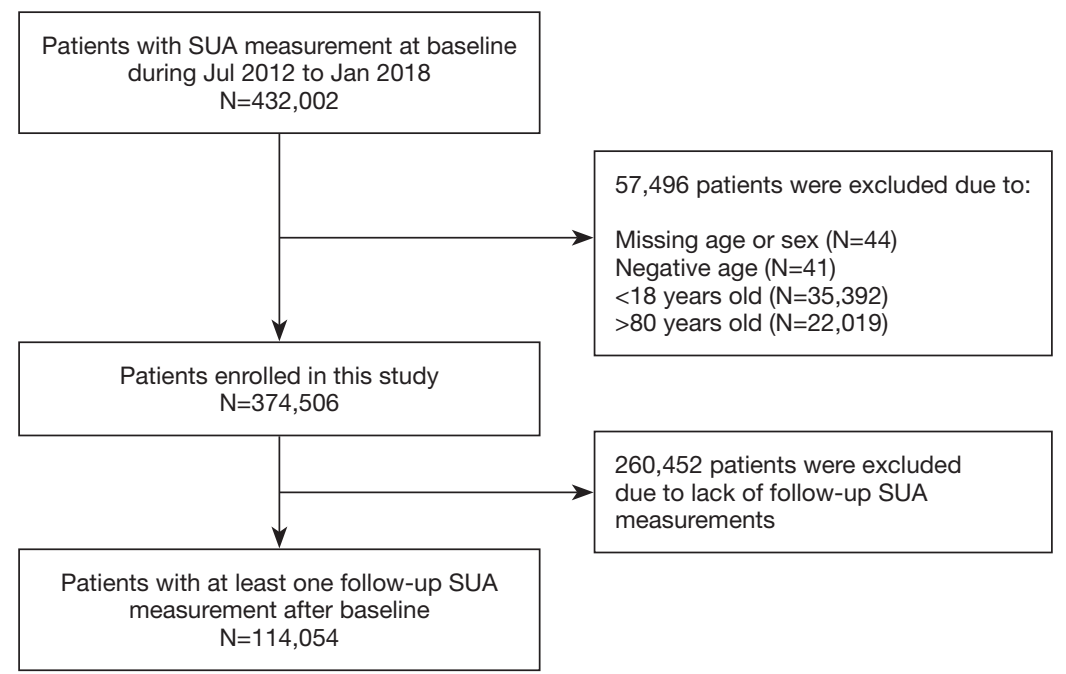

Figure 1 Population screening scheme.

patients with a specific disease and the whole population enrolled in the study. Subgroup population was defined as the denominator for the subgroup analysis. Odds ratios (ORs) were calculated for the association analysis between UA levels and related diseases. The definitions of diseases are as follows:

* Dyslipidemia: patients with diagnoses of dyslipidemia, or those prescribed lipid-lowering medication, or those with a total cholesterol (TC) level $\geq 5.72 \mathrm{mmol} / \mathrm{L}$, or a TG level $>1.70 \mathrm{mmol} / \mathrm{L}$, or a high-density lipoprotein (HDL-C) level $<0.91 \mathrm{mmol} / \mathrm{L}$;

* Type 2 diabetes mellitus (T2DM): patients with a prescribed intake of hypoglycemic agents or insulin, or serum with a $\mathrm{Hb} 1 \mathrm{Ac}$ level $\geq 6.5 \%$, or a fasting blood glucose level $\geq 7 \mathrm{mmol} / \mathrm{L}$, or a 2 -hour postprandial glucose level $\geq 11.1 \mathrm{mmol} / \mathrm{L}$ (24), excluding those with type 1 diabetes mellitus;

* Hypertension: Patients with diagnoses of stroke, coronary arterial disease or heart failure (who were subsumed under the cardio-cerebrovascular cases category), hypertension, or with a systolic blood pressure $(\mathrm{SBP}) \geq 140 \mathrm{mmHg}$, or a diastolic blood pressure (DBP) $\geq 90 \mathrm{mmHg}(25)$;

* Chronic kidney disease (CKD): patients with diagnoses of $\mathrm{CKD}$, or a kidney disease as diagnosed at the index visit and at an earlier visit at least 90 days before the index visit;

* Kidney and ureter calculus: patients with diagnoses of calculus of the kidney and ureter.

\section{Data sources}

The data used in this study are from the HISs of 4 tertiary hospitals.

\section{Bias}

We included the related factors (age, center, hypertension, glucose TG, HDL-C, and LDL-C) into the model to adjust for the potential bias.

\section{Study size}

The sample size in this study was determined by applying screening criteria in real-world settings.

\section{Outliers}

For the data of measurements, including Hb1Ac level, glucose level, TC, TG, HDL-C, SBP, DBP, and UA level, no outliers were found.

\section{Statistical methods}

The data are reported as mean \pm standard deviation for the normally distributed continuous variables, median and interquartile range for the non-normally distributed continuous variables, and proportions for the categorical variables. Differences between any 2 groups were compared 
using a $t$-test for the normally distributed continuous variables, a Wilcoxon test for the non-normally distributed continuous variables, and a Chi-squared test or Fisher test for the categorical variables. The Chi-squared test was also used to examine trends in proportions across groups with different UA levels. A Pearson or Spearman correlation analysis was used to examine the correlations among the continuous variables and uric acid levels. A multivariate binary logistic regression analysis was used to analyze associations among UA levels and dyslipidemia, T2DM, cardio-cerebral vascular disease, and CKD adjusting for demographic and clinical features. ORs and 95\% confidence intervals were calculated. A multivariate linear regression analysis was conducted to assess the linear associations among UA and biochemical parameters both as continuous variables.

\section{Results}

Of the 374,506 patients, $49.7 \%$ were male, and $50.3 \%$ were female. The mean age at the time at which the investigation was conducted of the overall test group, male population, and female population was $51.53,51.62$ and 51.43 years, respectively. The overall prevalence of HUA and gout were $14.8 \%$ and $0.5 \%$, respectively, and there were no significant differences within different provinces. Notably, Significant differences were observed between the sexes. The prevalence was higher in males than in females $(17.6 \%$ vs. $12.0 \%, 0.8 \%$ vs. $0.1 \%$; both $\mathrm{P}<0.001)$. Male patients with elevated UA levels had a decreased mean age, HDL-C level, and estimated glomerular filtration rate (eGFR), but increased prevalence rates for gout and CKD (see Table 1). Females with elevated UA levels had a decreased mean HDL-C level and eGFR, but an increased mean C-reactive protein (CRP) level, and prevalence rates for diseases, including gout, composite and individual cardiocerebrovascular diseases, and CKD (see Table 2).

\section{Associations among $U A$ levels and metabolism-related diseases}

In relation to the lowest (normal) UA level male group, higher UA level groups had significantly increased adjusted ORs (aORs) for dyslipidemia and CKD, and a decreased aOR for T2DM. Male patients with a UA level $\geq 9.0 \mathrm{mg} / \mathrm{dL}$ were more likely to have cardio-cerebrovascular events than those with normal UA levels (see Table 3). The associations between UA levels and diseases were similar among females, except that female in higher UA level groups did not have a significantly different aOR for T2DM than those in the lowest UA level group (see Table 4).

\section{Associations among UA levels and metabolism-related biochemical measurements}

Among the male subjects, UA was found to be negatively associated with HDL-C, CRP, glucose, HbA1c, eGFR and SBP, but positively associated with TG, and low-density lipoprotein cholesterol (LDL-C) (see Table 5).

Among the female subjects, UA was found to be negatively associated with HDL-C, glucose, and eGFR, but positively associated with TC, TG, and LDL-C (see Table 5).

\section{Associations among UA level changes from the baseline and changes in metabolism-related biochemical measurements from the baseline}

We established a retrospective cohort comprising 114,054 participants with follow-up UA data. The median interval from the baseline assessment to the latest follow-up UA level assessment of patients in the cohort was 98.9 days. Changes in UA from the baseline were negatively correlated with changes in eGFR and HbAlc from the baseline $(\mathrm{r}=-0.319$ and -0.074 ; both $\mathrm{P}<0.001)$ and positively correlated with changes in blood glucose, TC, TG, LDL-C, (r=0.110, 0.144, 0.082, and 0.012 respectively; all $\mathrm{P}<0.05$ ), however, no correlation was found with HDL-C among males. After adjusting the covariates, changes in UA levels were found to be negatively correlated with changes in eGFR and HbA1c, and positively correlated with changes in TC, TG, LDL-C, and glucose (see Table 6 and Figures 2-8).

Among females, changes in UA from the baseline measurement were negatively correlated with changes in eGFR, HDL-C, and HbA1c from the baseline measurements ( $\mathrm{r}=-0.317,-0.025$, and -0.052 ; all $\mathrm{P}<0.001)$ and positively correlated with changes in TC, TG, and LDL-C ( $\mathrm{r}=0.094$, $0.147,0.073$ respectively; all $\mathrm{P}<0.001)$. After adjusting the covariates, changes in UA were found to be significantly negatively associated with changes in eGFR, HDL-C, and HbA1c, but positively correlated with changes in TC, TG, LDL-C, and glucose (see Table 6 and Figures 9-15).

\section{Discussion}

This research examined the prevalence of HUA in 
Table 1 Baseline characteristics of adult males by uric acid levels

\begin{tabular}{|c|c|c|c|c|c|}
\hline Characteristics & $\mathrm{UA}<7.0 \mathrm{mg} / \mathrm{dL}$ & $\begin{array}{c}7.0 \mathrm{mg} / \mathrm{dL} \leq \mathrm{UA} \\
<8.0 \mathrm{mg} / \mathrm{dL}\end{array}$ & $\begin{array}{c}8.0 \mathrm{mg} / \mathrm{dL} \leq \mathrm{UA} \\
<9.0 \mathrm{mg} / \mathrm{dL}\end{array}$ & $\mathrm{UA} \geq 9.0 \mathrm{mg} / \mathrm{dL}$ & $\mathrm{P}$ \\
\hline N (\% of all males) & $154,074(82.7 \%)$ & $16,681(9 \%)$ & $7,635(4.1 \%)$ & $7,917(4.2 \%)$ & \\
\hline Age (years) & $52.72 \pm 15.43$ & $47.69 \pm 15.85$ & $46.3 \pm 15.83$ & $43.77 \pm 16.77$ & $<0.001$ \\
\hline $\mathrm{TC}(\mathrm{mmol} / \mathrm{L})$ & $4.48 \pm 1.04$ & $4.75 \pm 1.09$ & $4.76 \pm 1.12$ & $4.61 \pm 1.22$ & $<0.001$ \\
\hline HDL-C (mmol/L) & $1.12 \pm 0.31$ & $1.07 \pm 0.28$ & $1.05 \pm 0.28$ & $1 \pm 0.31$ & $<0.001$ \\
\hline LDL-C (mmol/L) & $2.69 \pm 0.81$ & $2.84 \pm 0.85$ & $2.82 \pm 0.86$ & $2.71 \pm 0.91$ & $<0.001$ \\
\hline eGFR $\left(\mathrm{mL} / \mathrm{min} / 1.73 \mathrm{~m}^{2}\right)$ & $100.61 \pm 19.43$ & $94.95 \pm 25.85$ & $92.44 \pm 29.34$ & $90.82 \pm 35.19$ & $<0.001$ \\
\hline $\mathrm{SBP}(\mathrm{mmHg})$ & $130.97 \pm 18.43$ & $132.21 \pm 19.05$ & $132.72 \pm 21.34$ & $130.31 \pm 21.89$ & 0.09 \\
\hline $\mathrm{DBP}(\mathrm{mmHg})$ & $78.85 \pm 11.7$ & $80.64 \pm 12.37$ & $80.92 \pm 12.74$ & $79.72 \pm 13.52$ & $<0.001$ \\
\hline \multicolumn{6}{|l|}{ Comorbidities (\%) } \\
\hline Hyperuricemia & 0.4 & 100 & 100 & 100 & $<0.001$ \\
\hline Gout & 0.5 & 3.2 & 7.7 & 12.2 & $<0.001$ \\
\hline Dyslipidemia & 48.7 & 57.7 & 57.6 & 51 & $<0.001$ \\
\hline Heart failure & 7.7 & 7.3 & 8.4 & 10.9 & $<0.001$ \\
\hline Chronic kidney disease & 2.2 & 4.8 & 6.9 & 9.5 & $<0.001$ \\
\hline Calculus of kidney and ureter & 1.6 & 1.9 & 2.4 & 1.8 & $<0.001$ \\
\hline
\end{tabular}

CRP, C-reactive protein; DBP, diastolic blood pressure; eGFR, estimated glomerular filtration rate; HDL-C, high-density lipoprotein cholesterol; LDL-C, low-density lipoprotein cholesterol; SBP, systolic blood pressure; TC, total cholesterol; TG, triglycerides.

populations from 3 provinces. In light of the different characteristics between the sexes found in previous studies $(26,27)$, male and female subjects were analyzed separately. HUA was defined as $\mathrm{UA} \geq 7.0 \mathrm{mg} / \mathrm{dL}$ in males or $\geq 6.0 \mathrm{mg} / \mathrm{dL}$ in females. Using this criterion, the enrolled HUA subjects were subsequently subdivided into 3 groups based on the prescribed cut-off values, and a statistical analysis was carried out. Under the newly published guidelines for the diagnosis and management of HUA and gout in China (28), the UA cut-off value used to diagnose HUA has been redefined as $\geq 420 \mu \mathrm{mol} / \mathrm{L}(7 \mathrm{mg} / \mathrm{dL})$ in both sexes. However, in this retrospective study, we continued to use the former standard to ensure conformity with real-world clinical practice in China during the study period. As Table 1 shows, the percentages of cardio-cerebrovascular disease in the reference group were higher than those in other groups; 
Table 2 Baseline characteristics of adult females by uric acid levels

\begin{tabular}{|c|c|c|c|c|c|}
\hline Characteristics & $\mathrm{UA}<6.0 \mathrm{mg} / \mathrm{dL}$ & $\begin{array}{c}6.0 \mathrm{mg} / \mathrm{dL} \leq \mathrm{UA} \\
<7.0 \mathrm{mg} / \mathrm{dL}\end{array}$ & $\begin{array}{c}7.0 \mathrm{mg} / \mathrm{dL} \leq \mathrm{UA} \\
<8.0 \mathrm{mg} / \mathrm{dL}\end{array}$ & $\mathrm{UA} \geq 8.0 \mathrm{mg} / \mathrm{dL}$ & $P$ \\
\hline $\mathrm{N}$ (\% of all females) & $165,678(88 \%)$ & $12,512(6.6 \%)$ & $5,028(2.7 \%)$ & $4,981(2.6 \%)$ & \\
\hline Age (years) & $51.3 \pm 15.89$ & $53.7 \pm 17.18$ & $52.29 \pm 18.3$ & $49.22 \pm 19.36$ & 0.359 \\
\hline $\mathrm{TC}(\mathrm{mmol} / \mathrm{L})$ & $4.83 \pm 1.07$ & $5.07 \pm 1.16$ & $5.02 \pm 1.22$ & $4.76 \pm 1.33$ & $<0.001$ \\
\hline HDL-C (mmol/L) & $1.29 \pm 0.33$ & $1.2 \pm 0.32$ & $1.16 \pm 0.32$ & $1.09 \pm 0.39$ & $<0.001$ \\
\hline LDL-C (mmol/L) & $2.83 \pm 0.85$ & $3 \pm 0.9$ & $2.96 \pm 0.95$ & $2.79 \pm 1.00$ & $<0.001$ \\
\hline eGFR $\left(\mathrm{mL} / \mathrm{min} / 1.73 \mathrm{~m}^{2}\right)$ & $103.57 \pm 19.73$ & $90.37 \pm 28.32$ & $86.04 \pm 33.37$ & $83.47 \pm 38.28$ & $<0.001$ \\
\hline $\mathrm{SBP}(\mathrm{mmHg})$ & $127.68 \pm 18.86$ & $129.96 \pm 19.47$ & $130.39 \pm 21.4$ & $129.68 \pm 23.67$ & $<0.001$ \\
\hline $\mathrm{DBP}(\mathrm{mmHg})$ & $76.43 \pm 11.16$ & $77.83 \pm 11.86$ & $78.54 \pm 13.22$ & $76.57 \pm 13.94$ & $<0.001$ \\
\hline \multicolumn{6}{|l|}{ Comorbidities (\%) } \\
\hline Hyperuricemia & 0.1 & 100 & 100 & 100 & $<0.001$ \\
\hline Gout & 0.1 & 0.3 & 0.6 & 1.6 & $<0.001$ \\
\hline Dyslipidemia & 42.3 & 57.8 & 58.9 & 51 & $<0.001$ \\
\hline Heart failure & 7.5 & 11.3 & 13.4 & 17.4 & $<0.001$ \\
\hline Chronic kidney disease & 1.6 & 5.2 & 10 & 12.7 & $<0.001$ \\
\hline Calculus of kidney and ureter & 0.8 & 0.8 & 0.6 & 1.1 & 0.618 \\
\hline
\end{tabular}

CRP, C-reactive protein; DBP, diastolic blood pressure; eGFR, estimated glomerular filtration rate; HDL-C, high-density lipoprotein cholesterol; LDL-C, low-density lipoprotein cholesterol; SBP, systolic blood pressure; TC, total cholesterol; TG, triglycerides.

however, as the aOR was $>1$ (see Table 3) there might be reasons for this. First, the aOR for cardio-cerebrovascular disease in Table 3 was adjusted for age, center, hypertension, and glucose. Second, due to missing data, the population used for the multivariate logistic regression was a subset of the population for Table 1 .

Nationwide epidemiological data for HUA in China remains limited. Previous studies from different periods and regions have indicated that the prevalence of HUA continues to increase (5). Research has shown that the prevalence of HUA was $6.9-27.30 \%$ in men and $3.65-$ $15.33 \%$ in women $(22,29-31)$. In this study, the severity of HUA was assessed using UA levels. Consistent with the results of previous regional epidemiological studies, we found that the total prevalence of HUA was $17.6 \%$ in men and $12.0 \%$ in women. More than half of the patients 
Table 3 Associations between uric acid levels and metabolism-related diseases among adult males

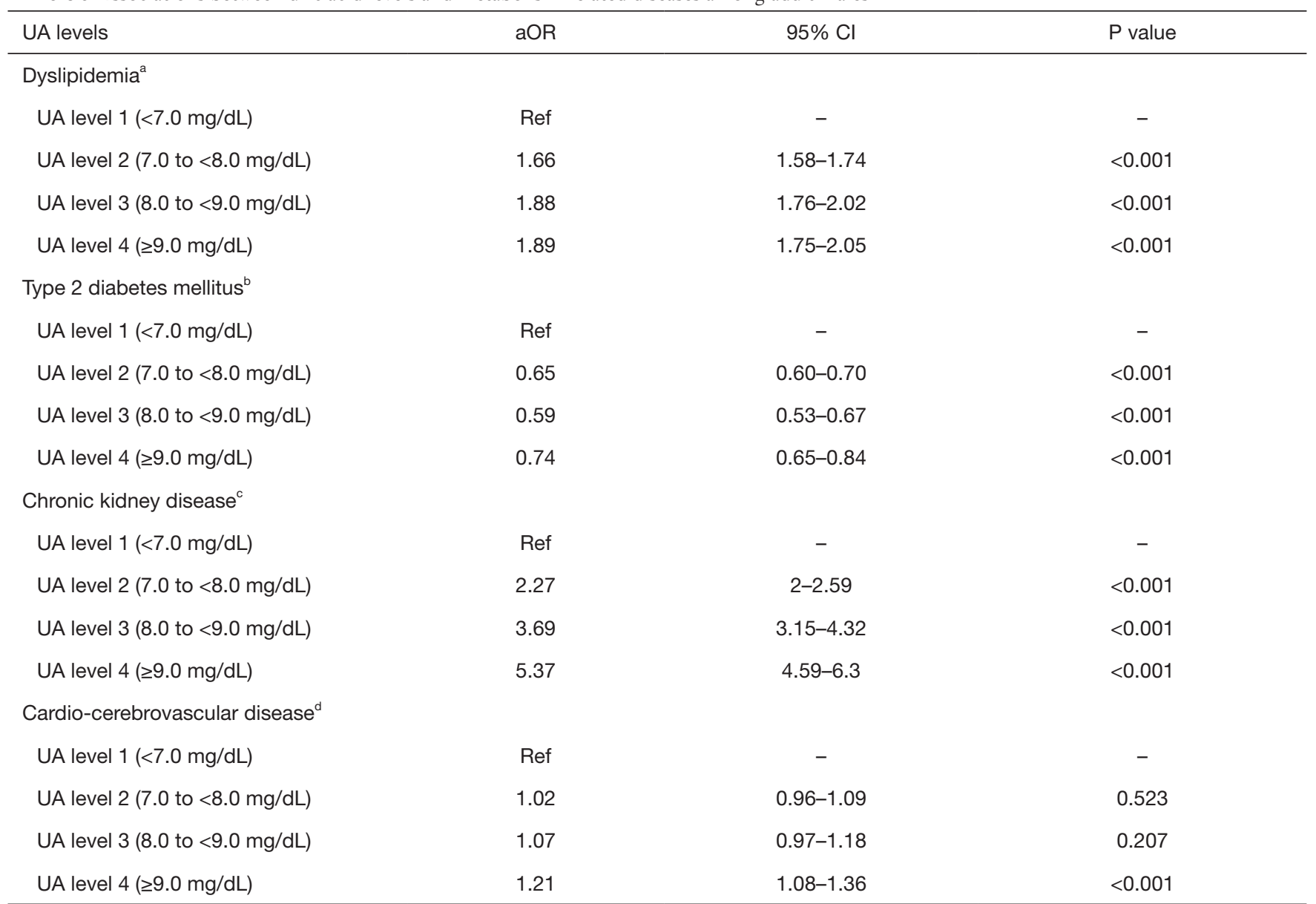

Among adult males, the number of dyslipidemia, type 2 diabetes mellitus, chronic kidney disease, cardio-cerebrovascular disease is $93,094,23,365,5,469,49,626$, respectively. ${ }^{a}$, adjusted for age, center, hypertension, and glucose; ${ }^{b}$, adjusted for age, center, hypertension, TG, HDL-C, and LDL-C; ' , adjusted for age, center, hypertension, TG, HDL-C, LDL-C and glucose; ${ }^{d}$, adjusted for age, center, hypertension, TG, HDL-C, LDL-C and glucose. CRP, C-reactive protein; DBP, diastolic blood pressure; eGFR, estimated glomerular filtration rate; HDL-C, high-density lipoprotein cholesterol; LDL-C, low-density lipoprotein cholesterol; SBP, systolic blood pressure; TC, total cholesterol; TG, triglycerides.

with HUA were categorized into the mild group (men: $7.0-8.0 \mathrm{mg} / \mathrm{dL}$, women: $6.0-7.0 \mathrm{mg} / \mathrm{dL}$ ). Female patients with HUA were more likely to be older than males and to be post-menopausal. The reason for this may be related to the change in estradiol (32), which is considered to play a protective role in regulating UA. Similar to our findings, a meta-analysis showed that the pooled prevalence of gout in the Chinese population was $1.1 \%$, and is remarkably higher in men $(1.5 \%)$ than women $(0.9 \%)(5)$.

HUA is often accompanied by hyperlipidemia. This may be because lipid metabolism disorders and uric acid metabolism share mutual influence mechanisms. Increased serum lipid, especially TG, is positively correlated with
UA (33), and elevated TG is an independent risk factor of HUA (34). Serum LDL, TC, and the ratio of TG to HDL are positively correlated with UA level, while HDL level is negatively correlated with UA level (35). In this study, we observed that TG, TC, and LDL increased as UA increased, while HDL was decreased. Consistent with previous studies, TG appeared to be more relevant than the other lipid indexes. Further, adults with HUA showed a higher possibility of concomitant dyslipidemia.

Previously, HUA was thought to be a predictor of insulin resistance and diabetes mellitus. HUA and insulin resistance can be interactive (36). Increased UA levels may be related to abnormal glucose tolerance, which may ultimately 
Table 4 Associations between uric acid levels and metabolism-related diseases among adult females

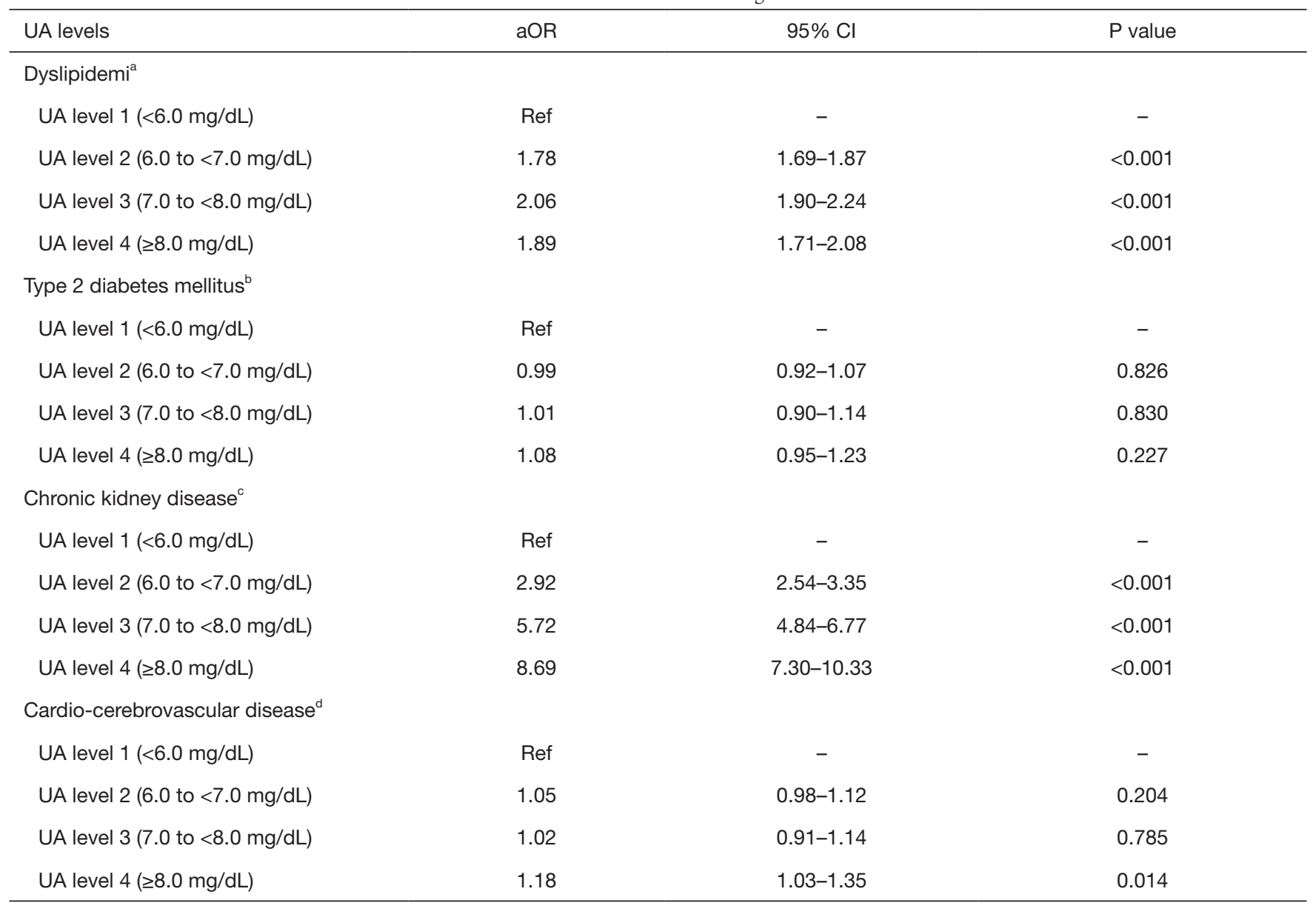

Among adult females, the number of dyslipidemia, type 2 diabetes mellitus, chronic kidney disease, cardio-cerebrovascular disease is $82,816,20,969,4,437,45,055$, respectively. ${ }^{a}$, adjusted for age, center, hypertension, and glucose; ${ }^{b}$, adjusted for age, center, hypertension, TG, HDL-C, and LDL-C; ' , adjusted for age, center, hypertension, TG, HDL-C, LDL-C and glucose; ${ }^{d}$, adjusted for age, center, hypertension, TG, HDL-C, LDL-C and glucose. CRP, C-reactive protein; DBP, diastolic blood pressure; eGFR, estimated glomerular filtration rate; HDL-C, high-density lipoprotein cholesterol; LDL-C, low-density lipoprotein cholesterol; SBP, systolic blood pressure; TC, total cholesterol; TG, triglycerides.

develop into diabetes mellitus (37). HUA with diabetes or abnormal glucose tolerance accounted for $31 \%$ to $55 \%$ (38). Similar to previous studies $(27,39)$, we found that UA levels were negatively correlated with blood glucose levels in both sexes at the baseline. This might be due to hyperfiltration caused by hyperglycemia, which can enhance the excretion of UA.

Emerging evidence suggests that HUA is associated with an increased risk of incidence and the progression of CKD (40). The results of previous studies on the association between UA with CKD in different sexes have been mixed (41-44). Despite lower concentrations of UA in females, the association between HUA and CKD in females was significantly stronger than that in males (23), demonstrated by eGFR measurement and CKD prevalence. The association between UA and CKD is consistent with our conclusion. Further, in our study, the prevalence of calculus of the kidney and ureter was associated with HUA in males, but this association was statistically non-significant in females.

Similar to our findings, epidemiological evidence supports an association between UA and the incidence of hypertension. Many observational studies have suggested that an increased risk of hypertension incidence may be independently caused by elevated UA levels (45-48). A previous study suggested that the risk of high blood 
Table 5 Correlation and coefficient between SUA levels' change and metabolism-related biochemical measurements' change among adult males and females (correlation analysis, $\triangle \mathrm{SUA}$ as independent variable)

\begin{tabular}{|c|c|c|c|c|c|c|}
\hline$\triangle S U A$ & \multicolumn{3}{|c|}{ Male } & \multicolumn{3}{|c|}{ Female } \\
\hline$\Delta \mathrm{TC}^{\mathrm{a}}$ & 0.001 & $-0.001-0.002$ & 0.273 & 0.003 & $0.001-0.005$ & $<0.001$ \\
\hline$\Delta T^{b}$ & 0.065 & $0.063-0.067$ & $<0.001$ & 0.064 & $0.061-0.067$ & $<0.001$ \\
\hline$\Delta \mathrm{HDL}-\mathrm{C}^{\mathrm{c}}$ & -0.007 & -0.008 to -0.006 & $<0.001$ & -0.013 & -0.014 to -0.012 & $<0.001$ \\
\hline$\triangle \mathrm{CRP}^{\mathrm{a}}$ & -1.593 & -1.983 to -1.202 & $<0.001$ & 0.171 & $-0.292-0.635$ & 0.468 \\
\hline$\Delta$ Glucose $^{e}$ & -0.111 & -0.119 to -0.103 & $<0.001$ & -0.045 & -0.054 to -0.036 & $<0.001$ \\
\hline$\triangle \mathrm{HbA} 1 \mathrm{c}^{\dagger}$ & -0.036 & -0.044 to -0.028 & $<0.001$ & -0.009 & -0.019 to -0.001 & 0.078 \\
\hline$\triangle \mathrm{eGFR}^{\mathrm{a}}$ & -4.202 & -4.276 to -4.128 & $<0.001$ & -4.81 & -4.894 to -4.727 & $<0.001$ \\
\hline
\end{tabular}

a, adjusted for age, center, hypertension, TG, HDL-C, LDL-C, glucose; ' , adjusted for age, center, hypertension, TC, HDL-C, LDL-C, glucose; ' , adjusted for age, center, hypertension, TC, TG, LDL-C, glucose; ${ }^{d}$, adjusted for age, center, hypertension, TC, TG, HDL-C, glucose; ', adjusted for age, center, hypertension, TG, HDL-C, LDL-C and DM treatment; ${ }^{\dagger}$, adjusted for age, center, hypertension, TG, HDL-C, LDL-C, glucose, and DM treatment. SUA, serum uric acid; CRP, C-reactive protein; DBP, diastolic blood pressure; eGFR, estimated glomerular filtration rate; HDL-C, high-density lipoprotein cholesterol; LDL-C, low-density lipoprotein cholesterol; SBP, systolic blood pressure; TC, total cholesterol; TG, triglycerides.

Table 6 Multivariate linear regression analysis of the association between change of serum uric acid from baseline and change of metabolismrelated biochemical measurements from baseline among males and females

\begin{tabular}{|c|c|c|c|c|c|c|}
\hline Variables & \multicolumn{3}{|c|}{ Male } & \multicolumn{3}{|c|}{ Female } \\
\hline eGFR $^{a}$ & -4.199 & -4.315 to -4.082 & $<0.001$ & -4.464 & -4.599 to -4.329 & $<0.001$ \\
\hline $\mathrm{TC}^{\mathrm{a}}$ & 0.053 & $0.046-0.061$ & $<0.001$ & 0.067 & $0.058-0.076$ & $<0.001$ \\
\hline $\mathrm{TG}^{\mathrm{b}}$ & 0.065 & $0.059-0.071$ & $<0.001$ & 0.078 & $0.071-0.085$ & $<0.001$ \\
\hline HDL-C ${ }^{d}$ & 0.001 & $-0.002-0.003$ & 0.607 & -0.005 & -0.008 to -0.002 & $<0.001$ \\
\hline Glucose $^{e}$ & 0.040 & $0.023-0.058$ & $<0.001$ & 0.037 & $0.017-0.056$ & $<0.001$ \\
\hline $\mathrm{HbA} 1 \mathrm{c}^{\mathrm{e}}$ & -0.035 & -0.052 to -0.018 & $<0.001$ & -0.030 & -0.048 to -0.011 & 0.002 \\
\hline
\end{tabular}

a , adjusted for sex, age, center, hypertension, TG, HDL-C, LDL-C, and glucose; ${ }^{b}$, adjusted for sex, age, center, hypertension, TC, HDL-C, LDL-C, and glucose; ' , adjusted for sex, age, center, hypertension, TC, TG, HDL-C, and glucose; ', adjusted for sex, age, center, hypertension, TC, TG, LDL-C, and glucose; ' , adjusted for sex, age, center, hypertension, TG, HDL-C, and LDL-C. CRP, C-reactive protein; DBP, diastolic blood pressure; eGFR, estimated glomerular filtration rate; HDL-C, high-density lipoprotein cholesterol; LDL-C, low-density lipoprotein cholesterol; SBP, systolic blood pressure; TC, total cholesterol; TG, triglycerides.

pressure in men with a UA level $>7.0 \mathrm{mg} / \mathrm{dL}$ was $80 \%$ higher than the risk at norm uricemia (49). Sex differences also exist. Notably, the prevalence of hypertension in HUA patients increased by 1.7 times in men and 3.4 times in women (50). We found a positive correlation between UA and hypertension both in SBP and DBP. Further, we found that this association was stronger in women than in men. This is probably because the average age of patients with 


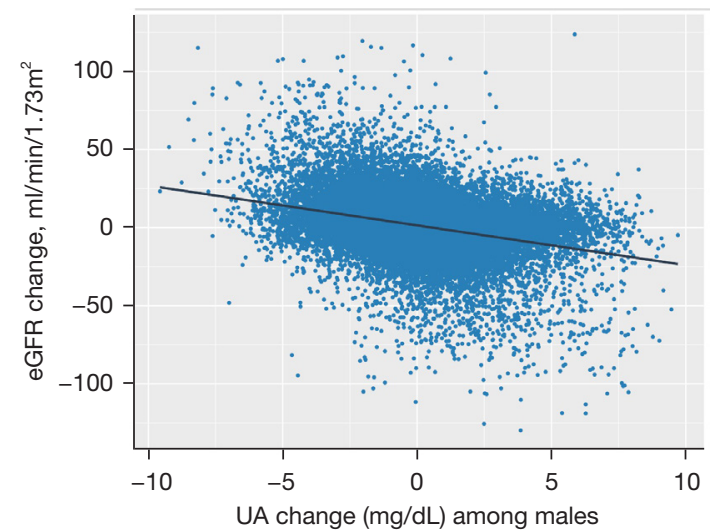

Figure 2 Correlation between changes in serum uric acid (UA) and changes in estimated glomerular filtration rate (eGFR) among males.

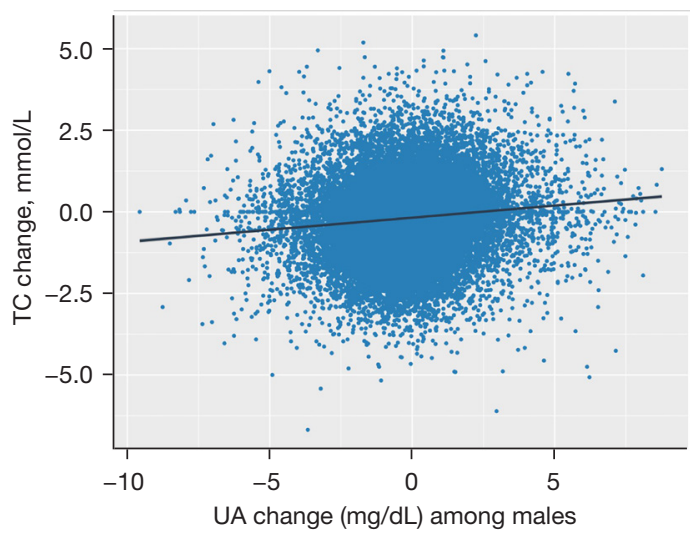

Figure 3 Correlation between changes in serum uric acid (UA) and changes in total cholesterol (TC) among males.

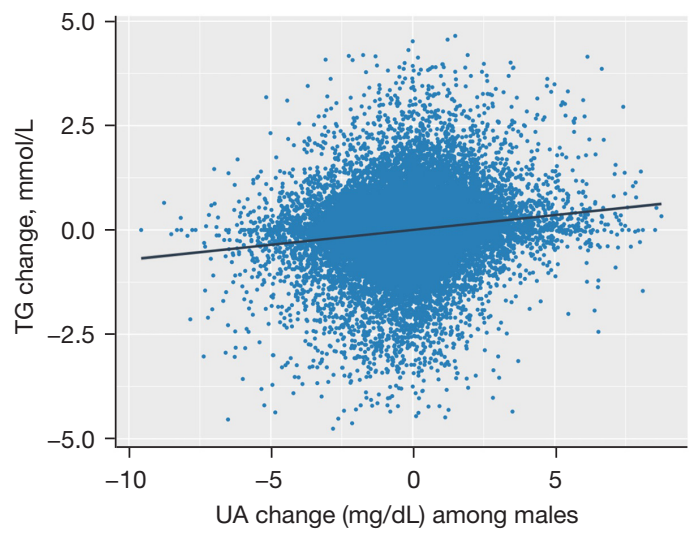

Figure 4 Correlation between changes in serum uric acid (UA) and changes in triglyceride (TG) among males.

(c) Annals of Translational Medicine. All rights reserved.

\section{Pang et al. HUA and its association with metabolic disorders}

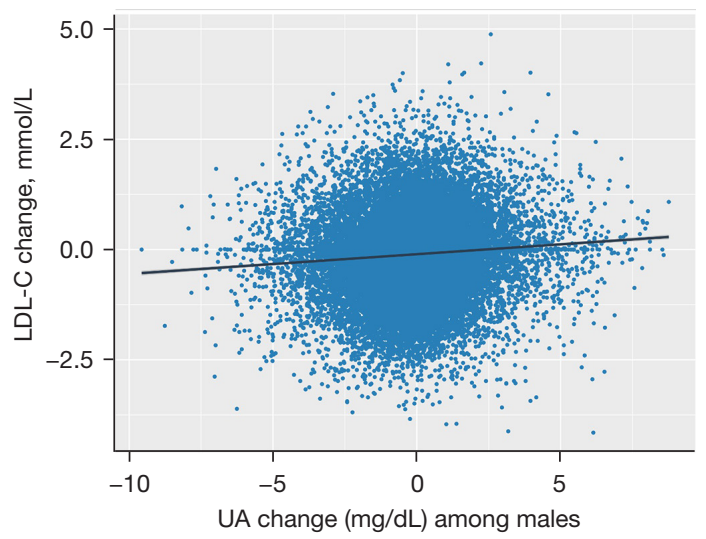

Figure 5 Correlation between changes in serum uric acid (UA) and changes in low-density lipoprotein cholesterol (LDL-C) among males.

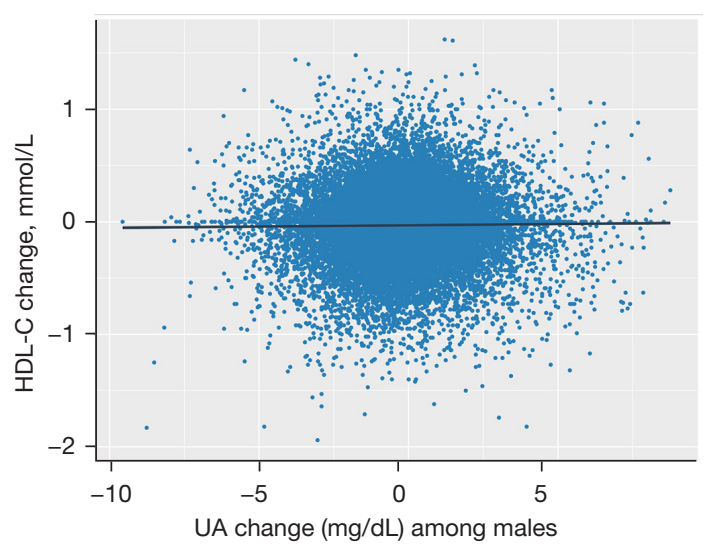

Figure 6 Correlation between changes in serum uric acid (UA) and changes in high-density lipoprotein cholesterol (HDL-C) among males.

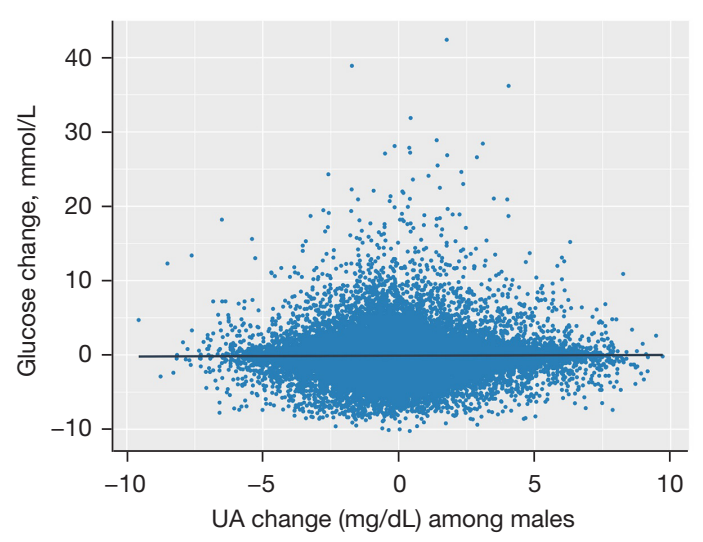

Figure 7 Correlation between changes in serum uric acid (UA) and changes in blood glucose among males. 


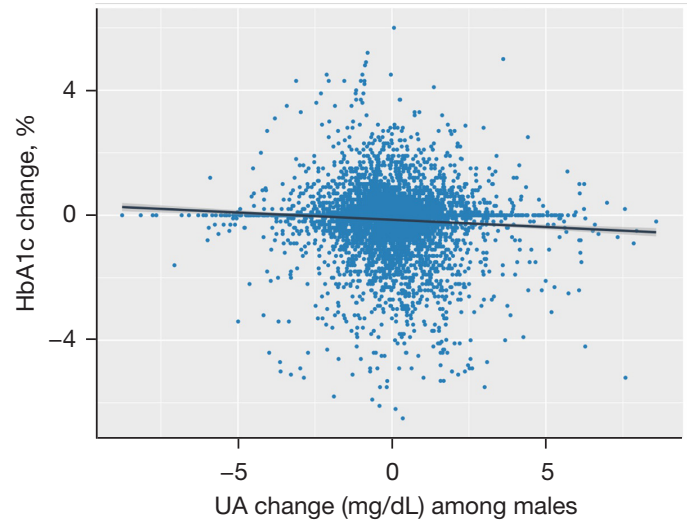

Figure 8 Correlation between changes in serum uric acid (UA) and changes in hemoglobin A1c (HbA1c) among males.

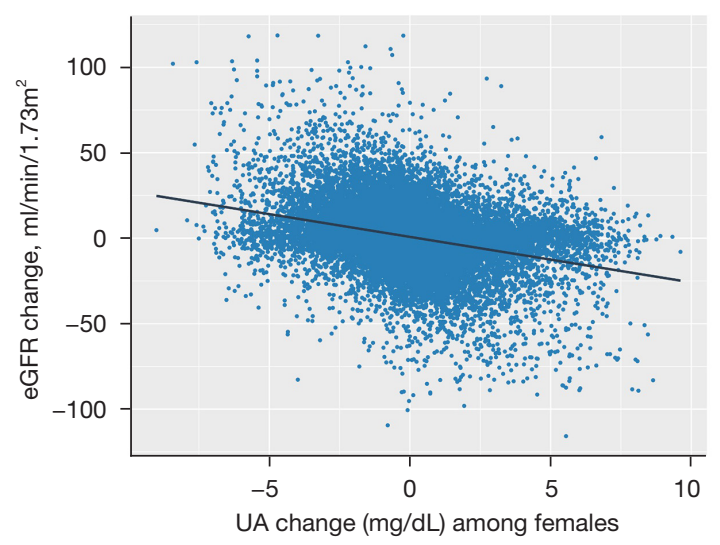

Figure 9 Correlation between changes in serum uric acid (UA) and changes in the estimated glomerular filtration rate (eGFR) among females.

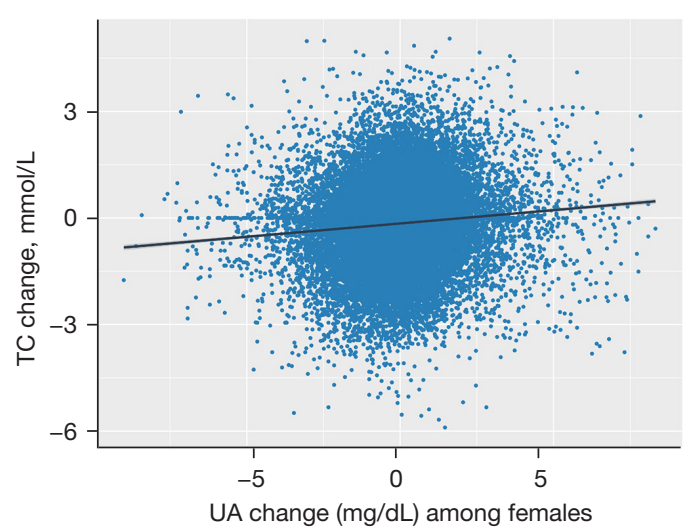

Figure 10 Correlation between changes in serum uric acid (UA) and changes in total cholesterol (TC) among females.

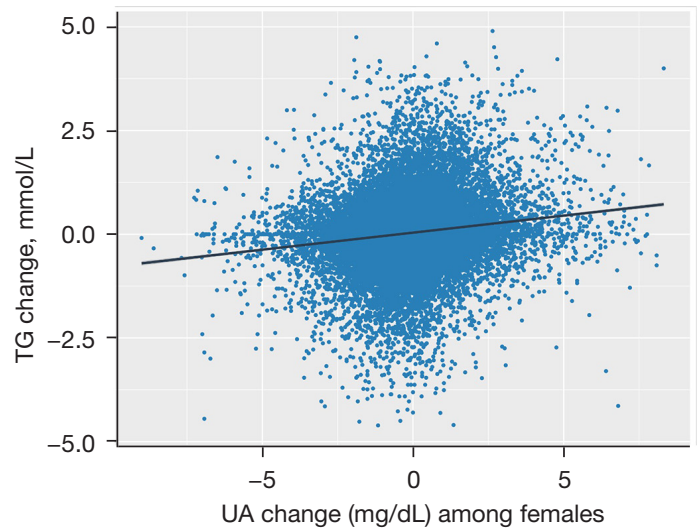

Figure 11 Correlation between changes in serum uric acid (UA) and changes in triglyceride (TG) among females.

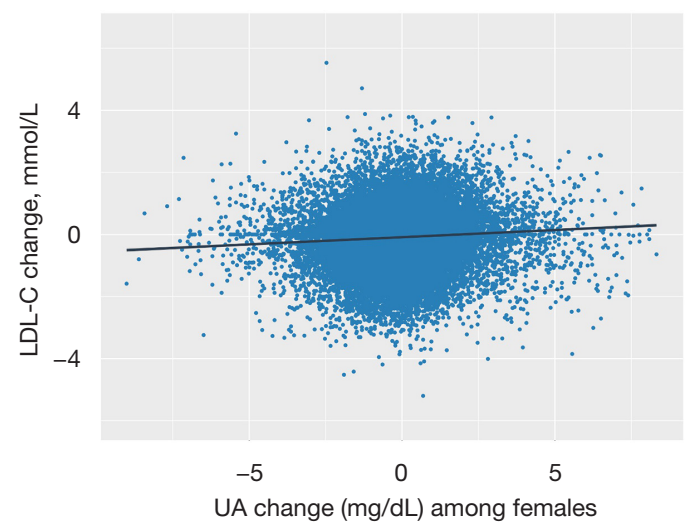

Figure 12 Correlation between changes in serum uric acid (UA) and changes in low-density lipoprotein cholesterol (LDL-C) among females.

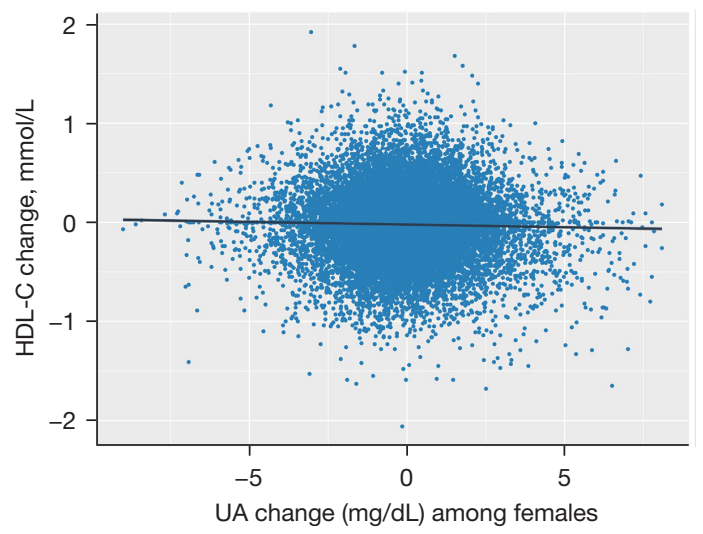

Figure 13 Correlation between changes in serum uric acid (UA) and changes in high-density lipoprotein cholesterol (HDL-C) among females. 


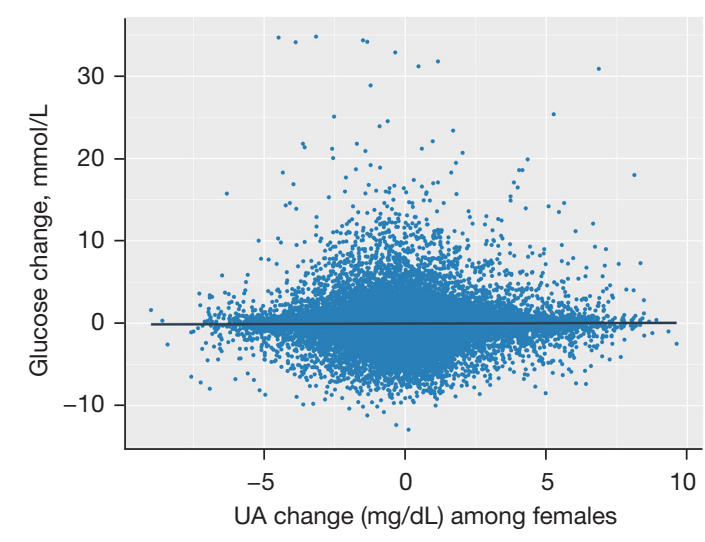

Figure 14 Correlation between changes in serum uric acid (UA) and changes in blood glucose among females.

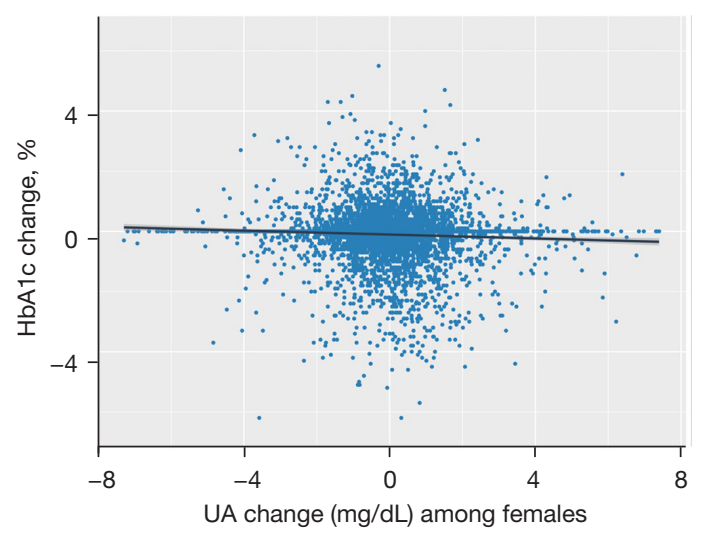

Figure 15 Correlation between changes in serum uric acid (UA) and changes in hemoglobin A1c (HbA1c) among females.

HUA is higher in women than man, and older people are at higher risk of hypertension.

HUA is a risk factor for cardiovascular events, development, and death (51). Our study showed that HUA is associated with a clustering of major CVD risk factors in the Chinese population. Further, we observed the prevalence of cardio-cerebrovascular diseases, including stroke, heart failure, and coronary arterial disease. As stated above, women with a higher age showed a stronger prevalence than men.

This study had several limitations. First, the relationship between UA and metabolism-related diseases was examined using cross-sectional data, and causality was not considered due to the lack of retrospective real-world data. Second, the retrospective real-world data were fully based on HIS and digital platforms, and the population enrolled were all subjects who sought healthcare services; thus, the overall population was not totally represented in the test group. Third, while we conducted an exploration of longitudinal data based on the retrospective cohort, the rate of loss in the follow-up data was high in the real-world setting. Finally, some potential confounders were not included in this study due to the data accessibility in the databases consulted, such as body weight, body mass index, insulin level and smoking and drinking history, resulted in the lack of subsequent analysis.

The prevention and treatment of uncertainties in real world study concludes a more principled approach to design and analysis in the presence of missing data. A careful design and conduct to limit the amount and impact of missing data.

In conclusion, we used large-scale retrospective HIS data to examine the sex-specific relationship among UA levels and metabolism-related diseases in Chinese patients. The sex-specific prevalence of HUA was observed in the Chinese population. The elevated prevalence of some metabolismrelated diseases might be associated with elevated UA levels in both male and female Chinese patients in real-world settings. Notably, both our cross-sectional and longitudinal results revealed that HUA was associated with dyslipidemia and CKD in both sexes.

\section{Acknowledgments}

Funding: This study was funded by the Key Technology Research and Development Program of Shandong (2016GSF201019) and the Jinan Science and Technology Bureau (201704116).

\section{Footnote}

Reporting Checklist: The authors have completed the STROBE reporting checklist. Available at https://dx.doi. org/10.21037/atm-21-5052

Data Sharing Statement: Available at https://dx.doi. org/10.21037/atm-21-5052

Conflicts of Interest: All authors have completed the ICMJE uniform disclosure form (available at https://dx.doi. org/10.21037/atm-21-5052). All authors reported that this study was funded by the Key Technology Research and Development Program of Shandong (2016GSF201019) and the Jinan Science and Technology Bureau (201704116). YL, 
YZ, and JL reported that they are from Jiangsu Hengrui Pharmaceuticals Co., Ltd. XY and TC reported that they are from Shanghai Palan DataRx Co., Ltd. FZ reported that he is from Shandong Health Medical Big Data Co., Ltd. The authors have no other conflicts of interest to declare.

Ethical Statement: The authors are accountable for all aspects of the work in ensuring that questions related to the accuracy or integrity of any part of the work are appropriately investigated and resolved. All procedures performed in this study involving human participants were in accordance with the Declaration of Helsinki (as revised in 2013). The study was approved by committee ethics board of Jinan Central Hospital, Cheeloo College of Medicine, Shandong University, Jinan, China (No. 2020-026-01). Individual consent for this retrospective analysis was waived.

Open Access Statement: This is an Open Access article distributed in accordance with the Creative Commons Attribution-NonCommercial-NoDerivs 4.0 International License (CC BY-NC-ND 4.0), which permits the noncommercial replication and distribution of the article with the strict proviso that no changes or edits are made and the original work is properly cited (including links to both the formal publication through the relevant DOI and the license). See: https://creativecommons.org/licenses/by-nc-nd/4.0/.

\section{References}

1. Neogi T. Clinical practice. Gout. N Engl J Med 2011;364:443-52.

2. Choi HK, Atkinson K, Karlson EW, et al. Purine-rich foods, dairy and protein intake, and the risk of gout in men. N Engl J Med 2004;350:1093-103.

3. Choi HK, Atkinson K, Karlson EW, et al. Alcohol intake and risk of incident gout in men: a prospective study. Lancet 2004;363:1277-81.

4. Wallace KL, Riedel AA, Joseph-Ridge N, et al. Increasing prevalence of gout and hyperuricemia over 10 years among older adults in a managed care population. J Rheumatol 2004;31:1582-7.

5. Liu R, Han C, Wu D, et al. Prevalence of Hyperuricemia and Gout in Mainland China from 2000 to 2014: A Systematic Review and Meta-Analysis. Biomed Res Int 2015;2015:762820.

6. Wu J, Qiu L, Cheng XQ, et al. Hyperuricemia and clustering of cardiovascular risk factors in the Chinese adult population. Sci Rep 2017;7:5456.
7. Borghi C, Agabiti-Rosei E, Johnson RJ, et al. Hyperuricaemia and gout in cardiovascular, metabolic and kidney disease. Eur J Intern Med 2020;80:1-11.

8. Mehrpour M, Khuzan M, Najimi N, et al. Serum uric acid level in acute stroke patients. Med J Islam Repub Iran 2012;26:66-72.

9. Lippi G, Montagnana M, Luca Salvagno G, et al. Epidemiological association between uric acid concentration in plasma, lipoprotein(a), and the traditional lipid profile. Clin Cardiol 2010;33:E76-80.

10. Alikor CA, Emem-Chioma PC, Odia OJ. Prevalence of hyperuricaemia in a rural population of Nigerian Niger Delta region. Niger J Med 2013;22:187-92.

11. Xu J, Peng H, Ma Q, et al. Associations of non-high density lipoprotein cholesterol and traditional blood lipid profiles with hyperuricemia among middle-aged and elderly Chinese people: a community-based cross-sectional study. Lipids Health Dis 2014;13:117.

12. Bhole V, Choi JW, Kim SW, et al. Serum uric acid levels and the risk of type 2 diabetes: a prospective study. Am J Med 2010;123:957-61.

13. Liu J, Tao L, Zhao Z, et al. Two-Year Changes in Hyperuricemia and Risk of Diabetes: A FiveYear Prospective Cohort Study. J Diabetes Res 2018;2018:6905720.

14. Meng J, Li Y, Yuan X, et al. Effects of febuxostat on insulin resistance and expression of high-sensitivity C-reactive protein in patients with primary gout. Rheumatol Int 2017;37:299-303.

15. Wei F, Chang B, Yang X, et al. Serum Uric Acid Levels were Dynamically Coupled with Hemoglobin A1c in the Development of Type 2 Diabetes. Sci Rep 2016;6:28549.

16. $\mathrm{Lu} \mathrm{ZS,} \mathrm{Lu} \mathrm{ZH,} \mathrm{Lu} \mathrm{H}$, et al. Association between hyperuricemia and hypertension in a Chinese population at a high risk of hypertension. Blood Press 2009;18:268-72.

17. Holme I, Aastveit AH, Hammar N, et al. Uric acid and risk of myocardial infarction, stroke and congestive heart failure in 417,734 men and women in the Apolipoprotein MOrtality RISk study (AMORIS). J Intern Med 2009;266:558-70.

18. Huang H, Huang B, Li Y, et al. Uric acid and risk of heart failure: a systematic review and meta-analysis. Eur J Heart Fail 2014;16:15-24.

19. Odden MC, Amadu AR, Smit E, et al. Uric acid levels, kidney function, and cardiovascular mortality in US adults: National Health and Nutrition Examination Survey (NHANES) 1988-1994 and 1999-2002. Am J Kidney Dis 2014;64:550-7. 


\section{Page 14 of 15}

20. Johnson RJ, Kang DH, Feig D, et al. Is there a pathogenetic role for uric acid in hypertension and cardiovascular and renal disease? Hypertension 2003;41:1183-90.

21. Wang SF, Shu L, Wang S, et al. Gender difference in the association of hyperuricemia with hypertension in a middle-aged Chinese population. Blood Press 2014;23:339-44.

22. Yu S, Yang H, Guo X, et al. Prevalence of hyperuricemia and its correlates in rural Northeast Chinese population: from lifestyle risk factors to metabolic comorbidities. Clin Rheumatol 2016;35:1207-15.

23. Kuwabara Y, Yasuno S, Kasahara M, et al. The association between uric acid levels and renal function of CKD patients with hyperlipidemia: a sub-analysis of the ASUCA trial. Clin Exp Nephrol 2020;24:420-6.

24. American Diabetes Association. Diagnosis and classification of diabetes mellitus. Diabetes Care 2010;33 Suppl 1:S62-9.

25. 1999 World Health Organization-International Society of Hypertension Guidelines for the Management of Hypertension. Guidelines Subcommittee. J Hypertens 1999;17:151-83.

26. Yang $Y$, Zhou W, Wang $Y$, et al. Gender-specific association between uric acid level and chronic kidney disease in the elderly health checkup population in China. Ren Fail 2019;41:197-203.

27. Tao M, Pi X, Ma X, et al. Relationship between serum uric acid and clustering of cardiovascular disease risk factors and renal disorders among Shanghai population: a multicentre and cross-sectional study. BMJ Open 2019;9:e025453.

28. Chinese Society of Endocrinology, Chinese Medical Association. Guideline for the diagnosis and management of hyperuricemia and gout in China (2019). Chinese Journal of Endocrinology and Metabolism 2020;36:1-13.

29. Zheng M, Lai Y, He X, et al. Correlation of detection of hyperuricemia with hypertension in healthy population in Guangzhou City. China Tropical Medicine 2008;8:512-4.

30. Chen S, Du H, Wang Y, et al. The epidemiology study of hyperuricemia and gout in a community population of Huangpu District in Shanghai. Chin Med J (Engl) 1998;111:228-30.

31. Cao Y, Liu Y, Li T, et al. Investigation of the increasing of policemen in blood uric acid and Triglyceride in Haikou. Hainan Medical Journal 2010;21:120-1.

32. Ichikawa A, Sumino H, Ogawa T, et al. Effects of longterm transdermal hormone replacement therapy on the

\section{Pang et al. HUA and its association with metabolic disorders}

renin-angiotensin- aldosterone system, plasma bradykinin levels and blood pressure in normotensive postmenopausal women. Geriatr Gerontol Int 2008;8:259-64.

33. Lai SW, Li TC, Ng KC. Hyperuricaemia and its related factors in Taiwanese middle-aged adults. Int J Nurs Pract 2002;8:56-60.

34. Ishizaka N, Ishizaka Y, Toda A, et al. Changes in waist circumference and body mass index in relation to changes in serum uric acid in Japanese individuals. J Rheumatol 2010;37:410-6.

35. Peng TC, Wang CC, Kao TW, et al. Relationship between hyperuricemia and lipid profiles in US adults. Biomed Res Int 2015;2015:127596.

36. Li C, Hsieh MC, Chang SJ. Metabolic syndrome, diabetes, and hyperuricemia. Curr Opin Rheumatol 2013;25:210-6.

37. Perez-Ruiz F, Aniel-Quiroga MA, Herrero-Beites AM, et al. Renal clearance of uric acid is linked to insulin resistance and lower excretion of sodium in gout patients. Rheumatol Int 2015;35:1519-24.

38. Puddu P, Puddu GM, Cravero E, et al. Relationships among hyperuricemia, endothelial dysfunction and cardiovascular disease: molecular mechanisms and clinical implications. J Cardiol 2012;59:235-42.

39. Li Q, Yang Z, Lu B, et al. Serum uric acid level and its association with metabolic syndrome and carotid atherosclerosis in patients with type 2 diabetes. Cardiovasc Diabetol 2011;10:72.

40. Mallat SG, Al Kattar S, Tanios BY, et al. Hyperuricemia, Hypertension, and Chronic Kidney Disease: an Emerging Association. Curr Hypertens Rep 2016;18:74.

41. Nagasawa Y, Yamamoto R, Shoji T, et al. Serum Uric Acid Level Predicts Progression of IgA Nephropathy in Females but Not in Males. PLoS One 2016;11:e0160828.

42. Li Z, Liu Q, Mao H, et al. Gender difference in the association of hyperuricemia with chronic kidney disease in southern China. Kidney Blood Press Res 2012;36:98-106.

43. Huang YP, Zheng T, Zhang DH, et al. Community-based study on elderly CKD subjects and the associated risk factors. Ren Fail 2016;38:1672-6.

44. Weiner DE, Tighiouart H, Elsayed EF, et al. Uric acid and incident kidney disease in the community. J Am Soc Nephrol 2008;19:1204-11.

45. Grayson PC, Kim SY, LaValley M, et al. Hyperuricemia and incident hypertension: a systematic review and metaanalysis. Arthritis Care Res (Hoboken) 2011;63:102-10.

46. Bombelli M, Ronchi I, Volpe $M$, et al. Prognostic value of serum uric acid: new-onset in and out-of-office hypertension and long-term mortality. J Hypertens 
2014;32:1237-44.

47. Krishnan E, Kwoh CK, Schumacher HR, et al. Hyperuricemia and incidence of hypertension among men without metabolic syndrome. Hypertension 2007;49:298-303.

48. Perlstein TS, Gumieniak O, Williams GH, et al. Uric acid and the development of hypertension: the normative aging study. Hypertension 2006;48:1031-6.

49. Ito $H$, Abe $M$, Mifune $M$, et al. Hyperuricemia is independently associated with coronary heart disease and renal dysfunction in patients with type 2 diabetes mellitus.
PLoS One 2011;6:e27817.

50. Kuwabara M, Niwa K, Nishi Y, et al. Relationship between serum uric acid levels and hypertension among Japanese individuals not treated for hyperuricemia and hypertension. Hypertens Res 2014;37:785-9.

51. Braga F, Pasqualetti S, Ferraro S, et al. Hyperuricemia as risk factor for coronary heart disease incidence and mortality in the general population: a systematic review and meta-analysis. Clin Chem Lab Med 2016;54:7-15.

(English Language Editor: L. Huleatt)

Cite this article as: Pang S, Jiang Q, Sun P, Li Y, Zhu Y, Liu J, Ye X, Chen T, Zhao F, Yang W. Hyperuricemia prevalence and its association with metabolic disorders: a multicenter retrospective real-world study in China. Ann Transl Med 2021;9(20):1550. doi: 10.21037/atm-21-5052 